\title{
The accuracy and robustness of plasma biomarker models for amyloid PET positivity
}

\author{
Andréa L. Benedet ${ }^{1,2^{*}+}$ (D, Wagner S. Brum ${ }^{1,3 \dagger}$, Oskar Hansson ${ }^{4,5}$, Alzheimer's Disease Neuroimaging \\ Initiative, Thomas K. Karikari 1,6, Eduardo R. Zimmer ${ }^{3,7,8}$, Henrik Zetterberg ${ }^{1,9,10,11,12}$, Kaj Blennow ${ }^{1,9}$ and \\ Nicholas J. Ashton 1,12,13,14,15
}

\begin{abstract}
Background: Plasma biomarkers for Alzheimer's disease (AD) have broad potential as screening tools in primary care and disease-modifying trials. Detecting elevated amyloid- $\beta$ (A $\beta$ ) pathology to support trial recruitment or initiating $A \beta$-targeting treatments would be of critical value. In this study, we aimed to examine the robustness of plasma biomarkers to detect elevated $A \beta$ pathology at different stages of the AD continuum. Beyond determining the best biomarker-or biomarker combination-for detecting this outcome, we also simulated increases in inter-assay coefficient of variability (CV) to account for external factors not considered by intra-assay variability. With this, we aimed to determine whether plasma biomarkers would maintain their accuracy if applied in a setting which anticipates higher variability (i.e., clinical routine).
\end{abstract}

Methods: We included 118 participants (cognitively unimpaired [CU, $n=50]$, cognitively impaired $[C l, n=68]$ ) from the ADNI study with a full plasma biomarker profile (Aß42/40, GFAP, p-tau181, NfL) and matched amyloid imaging. Initially, we investigated how simulated CV variations impacted single-biomarker discriminative performance of amyloid status. Then, we evaluated the predictive performance of models containing different biomarker combinations, based both on original and simulated measurements. Plasma A $\beta 42 / 40$ was represented by both immunoprecipitation mass spectrometry (IP-MS) and single molecule array (Simoa) methods in separate analyses. Model selection was based on a decision tree which incorporated Akaike information criterion value, likelihood ratio tests between the best-fitting models and, finally, and Schwartz's Bayesian information criterion.

Results: Increasing variation greatly impacted the performance of plasma $A \beta 42 / 40$ in discriminating $A \beta$ status. In contrast, the performance of plasma GFAP and p-tau181 remained stable with variations $>20 \%$. When biomarker models were compared, the models "AG" (Aß42/40 + GFAP; $A \cup C=86.5)$, "A" (A $342 / 40 ; A U C=82.3)$, and "AGP" $(A \beta 42 / 40+G F A P+p$-tau181; $A U C=93.5)$ were superior in determining $A \beta$ burden in all participants, within-CU, and

\footnotetext{
*Correspondence: andrea.benedet@gu.se

${ }^{\dagger}$ Andréa L. Benedet and Wagner S. Brum contributed equally to this work.

2 Translational Neuroimaging Laboratory, McGill Centre for Studies

in Aging, McGill University, Montreal, Quebec, Canada

Full list of author information is available at the end of the article

Data used in the preparation of this article were obtained from the

Alzheimer's Disease Neuroimaging Initiative (ADNI) database (http://adni.

loni.usc.edu/). As such, the investigators within the ADNI contributed to the design and implementation of ADNI and/or provided data but did not participate in analysis or writing of this report. A complete listing of ADNI investigators can be found at http://adni.loni.usc.edu/wp-ontent/ uploads/how_to_apply/ADNI_Acknowledgement_List.pdf.
} original author(s) and the source, provide a link to the Creative Commons licence, and indicate if changes were made. The images or other third party material in this article are included in the article's Creative Commons licence, unless indicated otherwise in a credit line to the material. If material is not included in the article's Creative Commons licence and your intended use is not permitted by statutory regulation or exceeds the permitted use, you will need to obtain permission directly from the copyright holder. To view a copy of this licence, visit http://creativecommons.org/licenses/by/4.0/. The Creative Commons Public Domain Dedication waiver (http://creativeco mmons.org/publicdomain/zero/1.0/) applies to the data made available in this article, unless otherwise stated in a credit line to the data. 
within-Cl groups, respectively. In the robustness analyses, when repeating model selection based on simulated measurements, models including IP-MS A $\beta 42 / 40$ were also most often selected. Simoa A $\beta 42 / 40$ did not contribute to any selected model when used as an immunoanalytical alternative to IP-MS Aß42/40.

Conclusions: Plasma A $342 / 40$, as quantified by IP-MS, shows high performance in determining $A \beta$ positivity at all stages of the AD continuum, with GFAP and p-tau181 further contributing at $\mathrm{Cl}$ stage. However, between-assay variations greatly impacted the performance of Aß42/40 but not that of GFAP and p-tau181. Therefore, when dealing with between-assay CVs that exceed 5\%, plasma GFAP and p-tau181 should be considered for a more robust determination of $\mathrm{A} \beta$ burden in $\mathrm{CU}$ and $\mathrm{Cl}$ participants, respectively.

Keywords: Amyloid, Plasma biomarker, Mass spectrometry, Immunoassay, Alzheimer's disease, ADNI, p-tau181, GFAP, $\mathrm{NfL}$

\section{Introduction}

Therapies targeting amyloid beta $(\mathrm{A} \beta)$, a defining feature in the pathophysiology of Alzheimer's disease (AD) [1], have recently been developed and proven to reduce $A \beta$ plaque load in the brain [2-5]. However, the cognitive benefit to symptomatic patients is either very mild or, in most cases, inconclusive. The reasons for these findings are unclear, but it is hypothesized that anti-A $\beta$ trials target a population too advanced in the disease course or that the trial duration does not have the length to observe a conclusive cognitive benefit. Nonetheless, therapeutic trials that target any phase of the $\mathrm{AD}$ continuum require confirmatory evidence of $A \beta$ burden - which is of principal importance in trials that will target preclinical $A D$. Cerebrospinal fluid (CSF) $A \beta 42 / 40$ and $A \beta$ positron emission tomography (PET) imaging are highly representative of $A \beta$ burden, and the latter is likely a fundamental obligation to prove target engagement throughout an intervention trial. Still, neither CSF nor PET biomarkers have the capacity to serve as a population screening tool for eligibility to anti-A $\beta$ trials.

A blood biomarker would act as a widely accessible and simplified triage of large and diverse populations to indicate appropriate individuals for therapeutic trial recruitment-irrespective of disease stage. Furthermore, in a clinical setting, an indication that mild cognitive symptoms are accompanied by $\mathrm{A} \beta$ pathology is of importance for the specialist delivering a diagnosis and symptomatic treatment and, soon, determining which disease-modifying treatment would be more suitable. The development of plasma biomarkers has been driven by targeting candidates proven to be successful in CSF. Novel mass spectrometry and ultra-sensitive immunoassay methods have recently allowed for the measurement of the $A \beta 42 / A \beta 40$ ratio and concentrations of phosphorylated tau (p-tau), glial fibrillary acidic protein (GFAP), and neurofilament light (NfL) in blood.

In this context, plasma $A \beta 42 / 40$ has been shown to be associated with CSF and PET measures of $A \beta$ and to be capable of identifying $A \beta$-positive individuals with high accuracy $[6,7]$. However, this is suggested to be assaydependent given the emerging data highlighting the superior accuracy of immunoprecipitation mass spectrometry (IP-MS) compared with ultrasensitive immunoassays for the detection of cerebral $A \beta$ [8]. In contrast, immunoassays for the detection of p-tau181 (as well as other epitopes; p-tau217 [9] and p-tau231 [10]) in plasma have been shown to be most valuable in identifying $A D$ in heterogeneous dementia population [11-14] and in predicting cognitive decline $[11,15,16]$, besides also being highly correlated with cerebral $A \beta$ burden. GFAP, a biomarker of astrocyte reactivity, increases in preclinical $\mathrm{AD}$ and is a promising plasma biomarker for this stage of the disease [17-19]. While CSF GFAP is seemingly associated with $A \beta$ pathology only in symptomatic individuals, plasma GFAP continues to rise during disease evolution in parallel with clinical syndrome severity and $A \beta$ accumulation $[17,19]$. These recent findings suggest that plasma GFAP is more closely related to abnormal $A \beta$ accumulation due to $A D$, whereas CSF GFAP may also incorporate changes independent of $A \beta$ pathology. Increases in plasma NfL are a widely reported finding in $\mathrm{AD}[20,21]$ and are also observed in pre-symptomatic familial $A D$ [22]. Contrasting to $A \beta$ and $p$-tau, NfL is not specific to AD pathology and is increased in many other neurodegenerative disorders [23] and acute neurological conditions [24]. Hence, plasma biomarkers for AD are either directly (A $342 / 40)$ or indirectly (e.g., tau phosphorylation, astrocyte reactivity and neurodegeneration) associated with presence of $A \beta$ pathology and could be used to indicate elevated $A \beta$ burden for therapeutic trials. They could be used as standalone tests or in a combinational biomarker panel, but different configurations and accuracies will likely depend on disease stage; A $\beta 42 / 40$ and GFAP are likely to be more associated with preclinical $A \beta$, whereas p-tau181 and NfL may be later markers with increases more apparent in the transition between preclinical and prodromal AD.

In this brief report, we studied the available plasma biomarker results from the Alzheimer Disease 
Neuroimaging Initiative (ADNI), A $\beta 42 / 40$, p-tau181, GFAP, and NfL, to suggest which biomarker(s) models would be best suited as a population prescreen for $A \beta$ burden in a clinically heterogeneous population (i.e., all participants independent of disease stage), composed by cognitively unimpaired (CU) participants and cognitively impaired (CI) patients. Further, we sought to determine the robustness of single or multi-biomarker models to identify $A \beta$ burden by assessing whether simulated changes in biomarker concentration $(0-20 \%)$ values would significantly impact on the predictive power or model selection.

\section{Methods}

\section{Study participants}

We used data from the multicenter ADNI study, designed to develop and validate neuroimaging and biochemical biomarkers for the early detection, monitoring, and treatment of $\mathrm{AD}$, and its inclusion criteria have been further described elsewhere [25]. All enrolled participants or authorized representatives provided informed consent, approved by ADNI center's respective Institutional Review Boards. For this study, we included participants based on the availability of A $\beta$ PET and full plasma biomarker profiles $[\mathrm{A} \beta 42 / 40$ (Washington UniversityIP-MS), p-tau181 (University of Gothenburg), GFAP (Simoa Neuro 4-plex E), and NfL (Simoa Neuro 4-plex E)]. Duplicate measurements of plasma biomarkers were excluded ( $n=9$ ), leading to a final sample of $n=118$ participants. Following ADNI's diagnostic criteria, subjects clinically classified as "control" were here named cognitively unimpaired (CU), whereas patients with mild cognitive impairment $(\mathrm{MCI})$ and dementia were here grouped into cognitively impaired (CI). Participants were classified for $\mathrm{A} \beta$-positivity based on having an abnormal $\mathrm{A} \beta$ PET scan, measured by $\left[{ }^{18} \mathrm{~F}\right]$-florbetapir PET, defined by a global cortical composite with standardized uptake value ratios (SUVr) with average value greater than 1.11 - a threshold that has been extensively validated to identify clinical and biologically relevant brain amyloidosis $[26,27]$.

\section{Plasma biomarker analysis}

For all plasma A $442 / 40$, GFAP, and NfL analyses, selected ADNI samples were collected within 3 months of an $A \beta$ PET scan; $n=130(50 \% \mathrm{~A} \beta+)$, cognitively normal $n=54$ $(37 \% \mathrm{~A} \beta+$ ), mild cognitive impairment $n=54 \quad(46 \%$ $\mathrm{A} \beta+)$, and $\mathrm{AD} n=22(91 \% \mathrm{~A} \beta+)$. ADNI blood samples are collected in $10 \mathrm{mLK} 2$-EDTA tubes and centrifuged within $1 \mathrm{~h}$ of collection at room temperature and centrifuged at $1300 \mathrm{~g}$ for $10 \mathrm{~min}$ to obtain the plasma fraction. All plasma samples were frozen on dry ice within $90 \mathrm{~min}$ of collection at ADNI sites, shipped to the Biomarker
Core laboratory, aliquoted into $0.5 \mathrm{~mL}$ polypropylene tubes, and stored at $-80^{\circ} \mathrm{C}$ (for detailed information see www.adni-info.org and adni.loni.usc.edu).

Plasma p-tau181 was measured on Simoa HD-X instruments (Quanterix, Billerica, MA, USA) in April 2020 at the Clinical Neurochemistry Laboratory, University of Gothenburg, Mölndal, Sweden [15]. To select the biomarker to represent the plasma $A \beta$ values, we initially compared a total of six plasma $A \beta 42 / 40$ measures: three mass spectrometry methods (Shimadzu, University of Gothenburg, Washington University) and three immunoassay methods [Simoa Neuro 4-plex E (Quanterix), Simoa $A \beta 40$ and $A \beta 42$ Advantage Kit, Elecsys Neuro Toolkit] analyzed between December 2020 to April 2021; samples were tested in a blinded fashion with analytical controls by the different laboratories (for detailed information on sample handling procedures, assay protocols, and performance, see www.adni-info.org and adni.loni.usc.edu). For this, we evaluated the plasma amyloid biomarkers' performance to predict $\mathrm{A} \beta$ PET positivity by comparing single biomarker-based receiver operating characteristics (ROC) curves with DeLong tests. The plasma A $\beta 42 / 40$ test with the highest area under the curve and the best performing commercially available assay were then selected for fitting logistic regression models in the next analysis stage.

\section{Statistical analysis}

Demographic information was compared between groups with $t$ tests for continuous variables and $X^{2}$ tests for categorical variables. Using a single-biomarker ROC curve approach, we compared the area under the curve (AUC) for each biomarker for cross-sectionally identifying patients with cerebral amyloidosis. To assess the robustness of these biomarkers individually, we repeated these analyses by introducing random variations in the original biomarker measurements, ranging from $1 \%$ to $20 \%$ in $\pm 1 \%$ intervals.

Next, we evaluated the power of different biomarker combinations to predict $A \beta$ positivity with a logistic regression framework. For this, an initial basic demographic model was built including only age, sex, and $A P O E-\varepsilon 4$ carriership status as predictors of $\mathrm{A} \beta$-positivity status. Then, we evaluated logistic regression models with the addition of the four biomarkers (A $\beta 42 / 40=$ "A", GFAP = "G", p-tau181= "P" and NfL="N") in all possible combinations: basic demographic model plus only one biomarker; basic demographic model plus different combinations of two or three biomarkers (e.g., AP; AGP); basic demographic model plus all four biomarkers (e.g., AGNP). To identify which specific biomarkers were the best predictors of brain amyloidosis, we evaluated models based on a decision tree, schematically represented 
in Fig. 2. Among all models, the best-fitting model was defined as the one with the lowest Akaike information criterion (AIC) value. Then, we performed likelihood ratio (LR) tests between the best-fitting model and those models with up to two AIC units above that of the best-fitting, leading to exclusion of models significantly inferior to the best-fitting model. Among the remaining models, the most useful biomarker combination was considered as the one present in the model with the lowest Schwartz's Bayesian information criterion (BIC), a more stringent metric than the AIC [28]. This process was repeated for logistic regression models fitted in three different populations across the $\mathrm{AD}$ continuum [all participants $(n=118$; also had CU/CI status as covariate in the model), $\mathrm{CU}(n=50)$, and $\mathrm{CI}(n=68)]$. This was firstly performed for IP-MS A $\$ 42 / 40$ from Washington University as "A $\mathrm{IP}-\mathrm{MS}$ ", and in an additional analysis, the Simoa Neuro 4-plex E A $\beta 42 / 40$ was utilized as the alternative " $\mathrm{A}$ Simoa" in the models.

For assessing the robustness of these model selections, we repeated these analyses by introducing random variations to the original biomarker measurements. Firstly, we tested robustness using the reported coefficients of variance $(\mathrm{CVs})$ for each analytical technique $\left[\mathrm{A}^{\mathrm{IP}-\mathrm{MS}}, \mathrm{CV}=4.0 \%^{7}\right.$; $\mathrm{p}$-tau $181^{15}, \mathrm{CV}=6.6 \%$; (Supplementary Table 1, NfL: $\mathrm{CV}=1.2 \%, \mathrm{~A}^{\text {Simoa }}, \mathrm{CV}=1.0 \%$; GFAP, $\mathrm{CV}=10.5 \%)$, and secondly, we tested robustness using random variations of $\pm 5 \%, \pm 10 \%, \pm 15 \%$, and $\pm 20 \%$ of the original biomarker values. Each robustness analysis was repeated in 10 iterations. We then applied the above-described decision tree to each iteration to assess whether the analytical variance could result in the selection of different biomarker combinations. Continuous predictors were centered and log-transformed depending on their distribution. All analyses were performed with R Statistical Software (https://www.r-proje ct.org/). Statistical significance was set as $\alpha=0.05$, and all tests were two-tailed.

\section{Results}

\section{Study participant characteristics}

The demographic characteristics of the study participants are displayed in Table 1 . In the full sample $(n=118)$, A $\beta$-positivity was confirmed by A $\beta$ PET in $n=60(50.8 \%)$ of individuals. $A \beta$-negative and $A \beta$-positive groups were evenly distributed for age, gender, and years of education. As expected, a significantly increased prevalence of patients with cognitive decline $(P<0.05), A P O E-\varepsilon 4$ carriage status $(P<0.01)$, and poorer MMSE $(P<0.0001)$ was observed in the $A \beta$-positive group.

\section{Comparison of plasma $A \beta 42 / 40$ methods to identify $A \beta$ PET burden}

Our first task was to select a plasma $A \beta 42 / 40$ method to represent " $A$ " in our models. Data from six plasma $A \beta 42 / 40$ assays were included in the ADNI database (Supplementary Figure 1). We determined that the IP-MS assay from Washington University discriminated $A \beta$-positive and $A \beta$-negative groups with

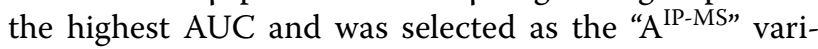
able in our models (AUC $=83.1 \%$; 95\% CI 75.5-90.7\%; Supplementary Figure 2A). This method was found to be statistically superior to the other five $A \beta 42 / 40$ assays included (DeLong test, Shimadzu, $P=0.007$;

Table 1 Demographics of selected participants from the ADNI cohort

\begin{tabular}{llll}
\hline & A $\boldsymbol{\beta}$ PET negative $(\boldsymbol{n = 5 8 )}$ & A $\boldsymbol{\beta}$ PET positive $(\boldsymbol{n}=\mathbf{6 0})$ & $\boldsymbol{P}$ value \\
\hline Age, years, median (IQR) & $70.8(66.5,75.7)$ & $73.8(69.9,77.4)$ & 0.14 \\
Clinical diagnosis, $n(\mathrm{CU} / \mathrm{Cl})$ & $30 / 28$ & $20 / 40$ & 0.04 \\
Female, $n(\%)$ & $24(41.4 \%)$ & $26(43.3 \%)$ & 0.98 \\
Years of education, median (IQR) & $18.0(14.2,18.0)$ & $16.0(14.0,18.0)$ & 0.33 \\
APOE- $\varepsilon 4$ carriers, $n(\%)$ & $15(25.9 \%)$ & $32(53.3 \%)$ & $<0.01$ \\
MMSE score, median (IQR) & $29.0(28.0,30.0)$ & $27.5(24.0,29.2)$ & $<0.0001$ \\
Florbetapir, global SUVR, median (IQR) & $1.00(0.954,1.03)$ & $1.33(1.22,1.46)$ & $<0.0001$ \\
A $\beta_{42 / 40}$ IP-MS, median (IQR) & $0.132(0.128,0.141)$ & $0.122(0.117,0.127)$ & $<0.0001$ \\
A $\beta_{42 / 40}$ Simoa, median (IQR) & $0.050(0.043,0.054)$ & $0.044(0.040,0.048)$ & $<0.01$ \\
GFAP, pg/mL, median (IQR) & $113(80.7,154)$ & $164(125,223)$ & $<0.001$ \\
P-tau181, pg/mL, median (IQR) & $11.7(8.2,17.2)$ & $18.8(13.1,23.0)$ & $<0.01$ \\
NfL, pg/mL, median (IQR) & $23.6(17.7,36.1)$ & $31.5(24.8,40.1)$ & 0.04 \\
\hline
\end{tabular}

Data shown as median (IQR; interquartile range) or $n$ (\%), as appropriate. Continuous variables were compared using $t$ test and Pearson's chi-square to compare frequencies of categorical variables between groups. As further explained, A $342 / 40$ IP-MS corresponds to the IP-MS assay from Washington University whilst A $342 / 40$ Simoa refers to the measurements from the Simoa Neuro 4-plex E assay

Abbreviations: $A \beta$ amyloid- $\beta, C U$ cognitively unimpaired, $C I$ mild cognitive impairment, MMSE Mini-Mental State Examination, $N f L$ neurofilament light chain, $P$-tau 181 tau phosphorylated at threonine 181, SD standard deviation, SUVR standardized uptake value ratio 
University of Gothenburg, $P=0.0006$; Simoa Neuro 4-plex E, $P=0.001$; Simoa $A \beta 40$ and $A \beta 42$ Advantage Kit, $P=0.0006$, Roche Elecsys, $P=0.03$ ). In addition, we aimed to have a commercially available immunoassay as an alternative "A" to run the sensitivity analysis. We thus compared A $442 / 40$ measured with Simoa Neuro 4-plex E (AUC $=65.1 \%$; 95\% CI 55.2-75.1\%) with A $\beta 42 / 40$ measured with Simoa $A \beta 40$ and $A \beta 42$ Advantage Kit (AUC $=55.3 \%$; 95\% CI 44.5-65.8\%). We found no statistical difference between these AUCs (DeLong test: $P=0.41$; Supplementary Figure $2 \mathrm{~B}$ ), and therefore, for practical reasons, we chose to perform the sensitivity analysis using $A \beta 42 / A \beta 40$ measured with Simoa Neuro 4-plex E "A Simoa", as GFAP and NfL are quantified in the same multiplex assay.

\section{Robustness of individual plasma biomarkers for A $\beta$-positivity}

We evaluated how well the biomarkers identify participants' $A \beta$ status (for biomarker distribution by $A \beta$ status see Supplementary Figure 3). All plasma biomarkers were significantly altered between $A \beta$-positive and $A \beta$-negative groups $\left(A \beta 42 / 40^{\mathrm{IP}-\mathrm{MS}}<0.0001\right.$; A $\beta 42 / 40^{\text {Simoa }}<0.01 ; \quad$ GFAP $<0.001 ; \quad$ p-tau $181<0.01$; $\mathrm{NfL}=0.04$ ). We then investigated how their AUC is affected by adding random variations on its original values. This robustness analysis sought to investigate if biomarkers' performance would remain constant if the values were to change within a given $\mathrm{CV}$. The rationale is that levels for plasma biomarkers may vary across analytical runs, laboratories, and cohorts [29], but data on this potential issue is essentially lacking for these biomarkers. This analysis was firstly done including all participants but also within CU and CI groups separately.

When all participants were evaluated, $\mathrm{A}^{\mathrm{IP}-\mathrm{MS}}$ had the highest AUC (AUC =83.1\%; 95\% CI 75.5-90.7\%), followed by GFAP (AUC $=71.7 \%$; 95\% CI 62.4-81.0\%) and p-tau181 (AUC=69.4\%; 95\% CI 59.6-79.3\%; Fig. 1A). However, with increased CV variation, the "predictive" power of $\mathrm{A}^{\mathrm{IP}-\mathrm{MS}}$ was drastically affected-while GFAP and p-tau181 AUCs remained stable through to a simulated $\mathrm{CV}$ of $20 \%$. Results from the CU group followed similar pattern to what was observed for the analysis with all participants (Fig. 1B). Differently, for the CI group, original biomarker values concluded that $\mathrm{A}^{\mathrm{IP}-\mathrm{MS}}$ have the highest AUC followed by p-tau181 and then

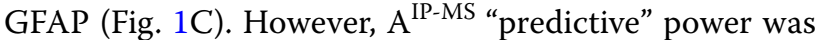
strongly impacted with even smaller variations on the $\mathrm{CV}$ $(<5 \%)$ as compared to what described in the analysis with all participants.

\section{Identifying A $\beta$-positivity using biomarker models}

The decision tree criteria for selecting biomarker models are illustrated in Fig. 2. This criterion firstly assessed models by AIC value and then LR tests between the bestfitting model and those models within two AIC units. Models significantly different from the best-fitting model were then rejected. Among the remaining models, the

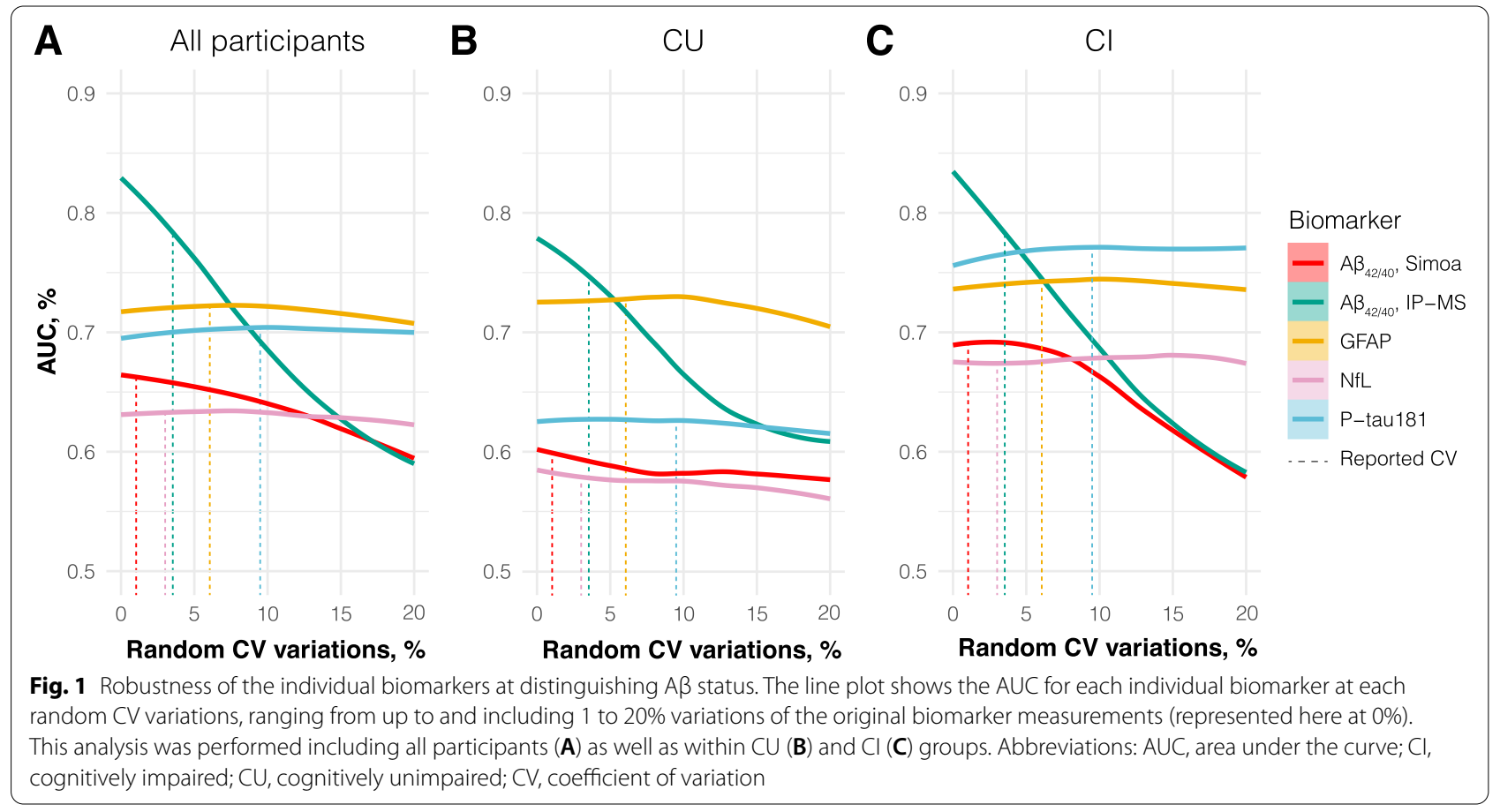




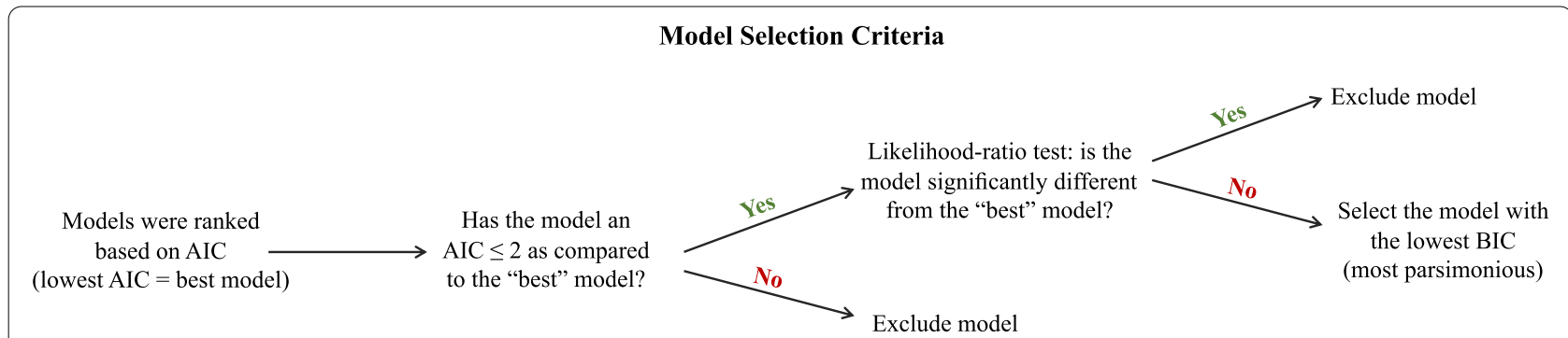

Fig. 2 Model selection criteria. The decision tree shows the steps that were followed when deciding the best biomarker model in each of the analyses. AIC, Akaike information criterion; BIC, Bayesian information criterion

most useful biomarker combination was considered as the one present in the model with the lowest BIC.

In all participants, the model "A ${ }^{\mathrm{IP}-\mathrm{MS}} \mathrm{G}$ " was selected as the superior model and demonstrated high accuracy for identifying $\mathrm{A} \beta$-positivity $(\mathrm{AUC}=86.5$; $95 \% \mathrm{CI} 79.7-$ 93.4\%; Table 2). Models "A ${ }^{\mathrm{IP}-\mathrm{MS}} \mathrm{GP}$ " and "A ${ }^{\mathrm{IP}-\mathrm{MS}} \mathrm{GN}$ " were $<2$ AIC units of the selected model and were not statistically different to "A ${ }^{\mathrm{IP}-\mathrm{MS}} \mathrm{G}$ " in LR tests $(P>0.5)$. However, these three-biomarker models were $>4$ BIC units away from "A ${ }^{\mathrm{IP}-\mathrm{MS}} \mathrm{G}$ ". The highest-ranking single biomarker model "A $\mathrm{IP}$-MS" was shown to be $>2$ AIC units from the selected model "A ${ }^{\mathrm{IP}-\mathrm{MS}} \mathrm{G}$ ". In CU participants, model "A IP-MS" was the model selected by our criteria $(\mathrm{AUC}=82.3 ;$ 95\% CI 68.5-96.1\%; Table 2). Models "A ${ }^{I P-M S} P^{2}$, "A ${ }^{I P-M S} N$ ", "A ${ }^{I P-M S} G$ ", and "A ${ }^{I P-M S} G N$ " were $<2$ AIC units of the selected model and were not statistically different to "A $\mathrm{IP}-\mathrm{MS}$ " in the LR test $(P>0.3)$. However, these biomarker models were $>2$ BIC units away

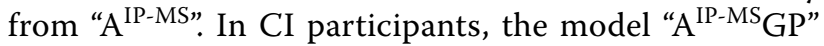
was selected by our criteria and demonstrated the highest overall accuracy for identifying $A \beta$-positivity in our study (AUC $=93.5 ; 95 \%$ CI 87.5-99.5\%; Table 2). Only the four-plasma biomarker model "A ${ }^{\mathrm{IP}-\mathrm{MS}} \mathrm{GNP}$ " was within 2 AIC units from the best model and was not statistically different to "A ${ }^{\mathrm{IP}-\mathrm{MS}} \mathrm{GP}$ " in LR test $(P=0.18)$. However, "A ${ }^{\mathrm{IP}-\mathrm{MS}} \mathrm{GNP}$ " was $>4$ BIC units away from "A ${ }^{\mathrm{IP}-\mathrm{MS}} \mathrm{GP}$ ". In summary, our criteria selected "A ${ }^{\mathrm{IP}-\mathrm{MS}} \mathrm{GP}$ ",

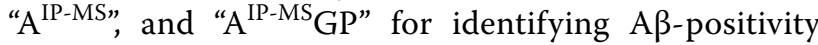
in all participants, $\mathrm{CU}$ participants, and $\mathrm{CI}$ patients, respectively.

We also performed the sensitivity analysis by replac-

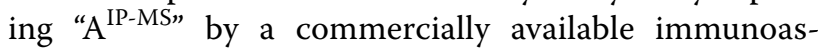
say for $A \beta 42 / 40$ " $\mathrm{A}$ Simoa", as previously described, which greatly impacted our results. In all participants, it was shown that "G" was the best model based on our criteria (Table 2). The model "G" had a modest accuracy for $A \beta$-positivity (AUC $=77.5$; 95\% CI 68.9-86.0). The AIC criteria ranked the model "GP" as the best fitted model and models defined as "G", "A ${ }^{\text {Simoa }} G$ ", "A ${ }^{\text {Simoa }} \mathrm{GP}$ ", and "GNP" were within 2 AIC units and not statistically different to "GP" in the LR tests $(P>0.05)$. However, the BIC favored the single biomarker model "G"

Table 2 Summary information of the selected biomarker models (original measurements)

\begin{tabular}{|c|c|c|c|c|c|c|c|c|}
\hline & Model & AIC & BIC & $R^{2}$, unadjusted & $R^{2}$, adjusted & AUC, $95 \% \mathrm{Cl}$ & LRT $x^{2}$ & $P$ value \\
\hline \multicolumn{9}{|l|}{ IP-MS for $A \beta 42 / 40$} \\
\hline All participants & $A G$ & 124.2 & 143.6 & $40.7 \%$ & $37.5 \%$ & $86.5 \%(79.7,93.4)$ & 53.3 & $<0.0001^{*}$ \\
\hline $\mathrm{CU}$ & A & 62.6 & 76.7 & $33.5 \%$ & $25.9 \%$ & $82.3 \%(68.5,96.1)$ & 16.7 & $<0.001^{*}$ \\
\hline $\mathrm{Cl}$ & AGP & 59.2 & 77.0 & $58.7 \%$ & $52.9 \%$ & $93.5 \%(87.5,99.5)$ & 43.5 & $<0.0001^{*}$ \\
\hline \multicolumn{9}{|c|}{ Simoa for $A \beta 42 / 40$} \\
\hline All participants & G & 145.5 & 159.4 & $22.2 \%$ & $19.4 \%$ & $77.5 \%(68.9,86.1)$ & 28.0 & $<0.0001^{*}$ \\
\hline $\mathrm{CU}$ & G & 71.9 & 81.4 & $11.0 \%$ & $3.1 \%$ & $72.8 \%(58.1,87.6)$ & 5.4 & 0.04 \\
\hline $\mathrm{Cl}$ & GP & 71.7 & 85.0 & $42.2 \%$ & $37.6 \%$ & $87.1 \%(78.4,95.9)$ & 32.4 & $<0.01^{*}$ \\
\hline \multicolumn{9}{|l|}{ Demographic } \\
\hline All participants & - & 155.6 & 166.7 & $12.7 \%$ & $10.5 \%$ & $70.7 \%(61.3,80.0)$ & - & - \\
\hline $\mathrm{CU}$ & - & 74.0 & 81.7 & $2.4 \%$ & $-4.0 \%$ & $58.1 \%(41.9,74.3)$ & - & - \\
\hline $\mathrm{Cl}$ & - & 78.9 & 87.8 & $27.8 \%$ & $24.5 \%$ & $81.3 \%(71.0,91.5)$ & - & - \\
\hline
\end{tabular}

Abbreviations: AIC Akaike information criterion, AUC area under the curve, BIC Bayesian information criterion, $C U$ cognitively unimpaired, $C l$ mild cognitive impairment, LRT likelihood ratio test

${ }^{*} P$ value of the likelihood ratio test comparing the selected model with the demographic model on the respective sample group 
(159.4) rather than "GP" (161.2) to be best fitting model. In CU participants, the model " $G$ " was also selected as the superior model for $A \beta$-positivity $(\mathrm{AUC}=72.8$; 95\% CI 58.1-87.6). Only "GN" was within 2 AIC units and was not statistically different to "G" $(P=0.29)$ but $>2$ BIC units from the best fitted model. In CI participants, the model "GP" was selected as the superior model A $\beta$-positivity (AUC=87.1; CI 78.4-95.9). Models "A ${ }^{\text {Simoa }}$ GP" and "GNP" were within 2 AIC units and were not statistically different to "GP" $(P>0.25)$. In summary, when using immunoassay instead of IP-MS determinations for "A", our criteria selected " $G$ " for predicting $A \beta$-positivity in all participants and $C U$ participants. The model "GP" was selected for predicting $\mathrm{A} \beta$-positivity in CI participants.

\section{Comparing selected models for $\mathrm{A} \boldsymbol{\beta}$-positivity}

We compared the selected models from each category (all participants, CU participants and CI participants)

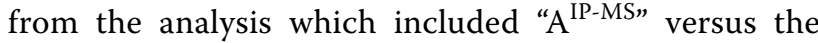
analysis which used immunoassays for " $A$ Simoa". In two scenarios (all participants and CI participants), mod-

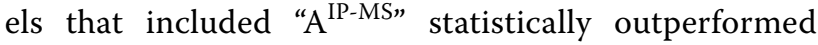
the equivalent analysis without; all participants (" $\mathrm{A}$ IP${ }^{M S} G$ " versus " $G$ ", $P=0.017$ ) and $C I$ participants ("A ${ }^{I P-}$ ${ }^{\mathrm{MS}} \mathrm{GP}$ " versus "GP", $P=0.042$ ). In CU participants, no

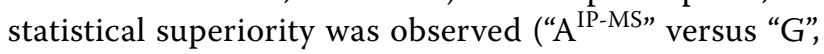
$P=0.20)$.

\section{Robustness of plasma biomarkers models for $\mathrm{A} \boldsymbol{\beta}$-positivity}

Next, after demonstrating that certain biomarkers models have superiority in determining $A \beta$ status at different stages of the disease, we sought to perform a robustness analysis to investigate if the selected models would remain constant if the biomarker values were to change within a given simulated $\mathrm{CV}$. We performed 10 iterations of randomly changed values for each one of the assays (A $\beta 42 / 40$ defined as a single assay). Firstly, we changed biomarker values within and up to the reported CV of each assay (see methods), and secondly, we changed biomarker values within and up to $5 \%, 10 \%$, $15 \%$, and $20 \%$-anticipating larger variations in multilaboratory comparisons. The same model selection decision tree (Fig. 2) was then applied to each robustness iteration.

Overall, the robustness of the reported variations did not largely impact on the model selection (Supplementary Table 1). However, if an increased variation up to $10 \%$ (or greater) was applied, the model selection shifted from "A IP-MS" to "G" in CU participants (Fig. 3A). Limited change in biomarker selection was seen for all and CI participants, with some deviation when $\mathrm{CV}$ varied at $15-20 \%$.

\section{Discussion}

In this study, our results denote that plasma $A \beta 42 / 40$ as determined by IP-MS was the best predictor of $\mathrm{A} \beta$-positivity, followed by $\mathrm{p}$-tau181 and GFAP. In a novel approach, preparing for such tests in clinical chemistry routine, we were interested in how variations in the biomarker measurements would impact the robustness of these biomarker performances. Random variations on the $\mathrm{CV}$ indicated that, around a simulated CV of $5 \%$, the accuracy of IP-MS A $\beta 42 / 40$ drops below to that of GFAP and p-tau181. In contrast, GFAP and p-tau181 performances remain stable even at a $20 \%$ $\mathrm{CV}$. When biomarkers were evaluated in several combinations of models, IP-MS A $\beta 42 / 40$ was the most significant contributor in predicting $A \beta$-positivity at the preclinical stages of AD, and adding $\mathrm{p}$-tau181, GFAP, or NfL did not significantly improve this finding. At the CI stages of the AD continuum, however, a model combing IP-MS A $\beta 42 / 40$, GFAP, and p-tau181 was found to be the best indicator of $A \beta$-positivity and results in very high accuracy. In general, models that included IP-MS A $342 / 40$ significantly outperformed model selections that included Simoa $A \beta 42 / 40$ as an alternative. We then investigated how the variations in biomarker $\mathrm{CV}$ would impact on the optimal model selection. With small variations in biomarker measurements, all selected models were preserved and shown to be robust. However, for CU participants, IP-MS measurements were not able to withstand a larger variation $(\mathrm{CV}>10 \%)$, being subsequently replaced by GFAP in the majority of model iterations. Originally selected $A \beta$-positivity models which included all participants and CI were robust, i.e., were most frequently selected, up to $15 \%$.

The use of plasma biomarkers to highlight underlying cerebral $A \beta$ pathology is greatly anticipated in clinical routine and disease-modifying trials, for both symptomatic and preclinical stages of AD. An increasing number of plasma biomarkers, shown to be related to $A \beta$ pathology, have now been reported [7, 15, 30,31], but it is yet to be determined which combinations are best suited in a heterogeneous population (e.g., diagnosis independent), preclinical or symptomatic stages. In this study, we show that IP-MS A $\beta 42 / 40$ have high accuracy in the detection of $A \beta$ pathology at all stages of the AD continuum and, in combination with GFAP and p-tau181, had a very high accuracy to determine $A \beta$-positivity in $\mathrm{CI}(>93 \%)$. There is a mixture of reports about the use plasma $A \beta 42 / 40$ in the literature [32]. While immunoassay results of p-tau from differing platforms are seemingly concordant with reproducible results and measures of plasma NfL and GFAP tend to utilize the same Simoa technology [33], methods to determine plasma $A \beta 42 / 40$ varies. This study shows the importance of method choice for the detection 


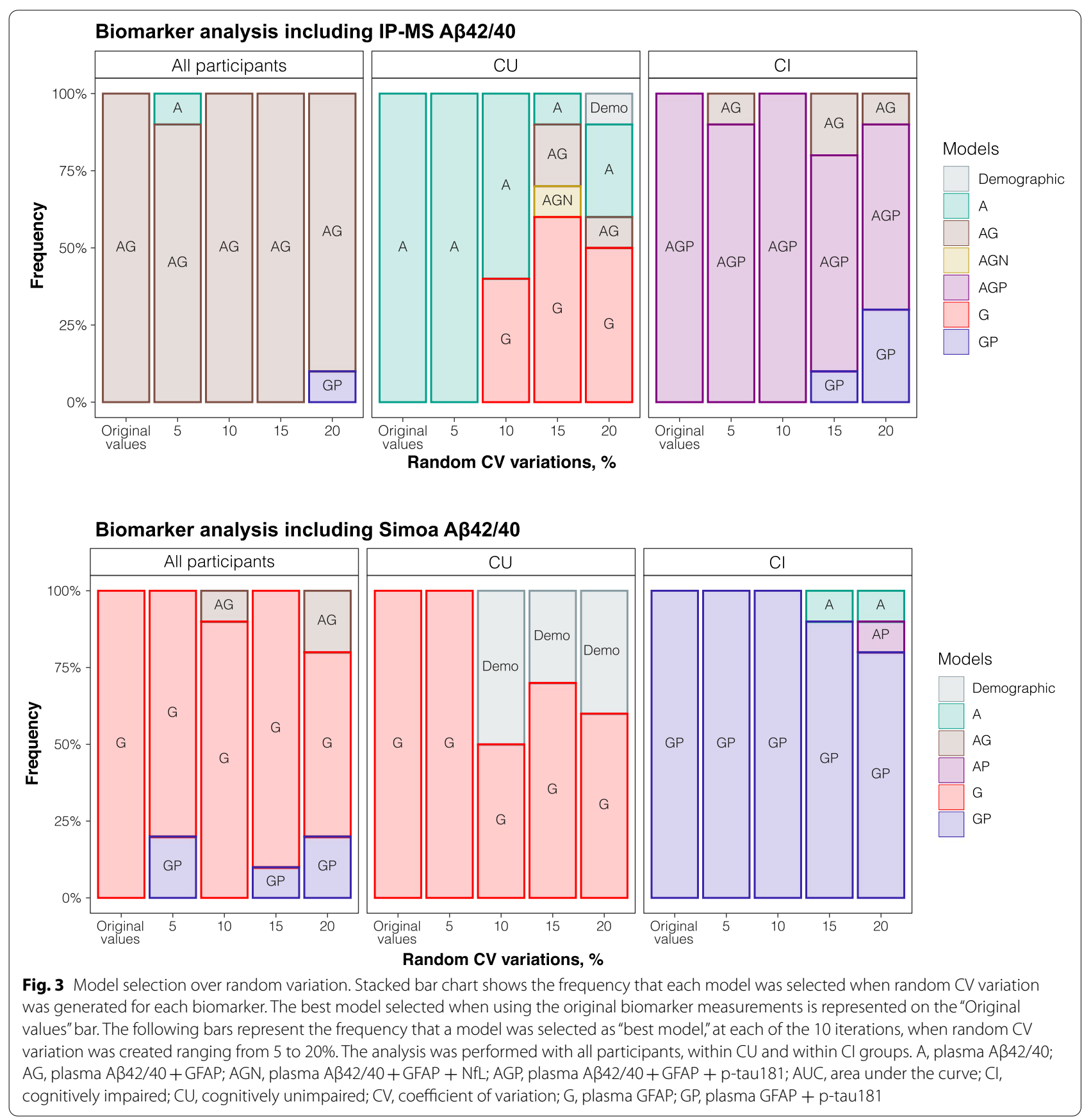

of brain amyloidosis by plasma $\mathrm{A} \beta$ since, when IP-MS measures of $A \beta 42 / 40$ were not included, $A \beta$-positivity was best represented by GFAP and p-tau181 and not by immunoassay determinations of $A \beta 42 / 40$. It is also important to signify that models that included IP-MS significantly outperformed models without it.

It is unlikely that $A \beta$ PET will be replaced from the recruitment process in anti-A $\beta$ trials, as target engagement and possible termination of $A \beta$ removal agents are necessary to determine participant's baseline and subsequent changes in $A \beta$ burden relative to the intervention process [4]. However, the plasma biomarker models demonstrated in this study may act, with good accuracy, as important initial screening tools to enrich a population for a larger success rate of A $\beta$ PET scan or tau PET scans [4] in the recruitment process. Our aim was to report the best plasma models for this process while acknowledging that IP-MS technology currently has constraints on 
availability and costs in comparison to semi-automated immunoassay methods. Thus, we included a commercially available immunoassay which did not significantly add to any biomarker model and was inferior to IP-MS A $342 / 40$, GFAP, and p-tau181 at the single biomarker level. Therefore, at this time, it is important to disseminate that IP-MS A $\beta 42 / 40$ measurements cannot simply be replaced by immunoassay $A \beta 42 / 40$ and, if IP-MS is not a viable option, $A \beta$-positivity is best represented by surrogate measures of $A \beta$ pathology, e.g., GFAP and $\mathrm{p}$-tau181, as shown in this study. This difference between A $\beta$ methods could be explained by IP-MS being less prone to matrix effects that are particularly noticeable in complex biological fluids such as blood.

However, there are constraints to $A \beta 42 / 40$ as a plasma biomarker which could be significant limiting factor in clinical chemistry routine. As $A \beta 42 / 40$ is suggested to change by only $10 \%$ in $\mathrm{A} \beta$-positivity individuals, compared with $50 \%$ in CSF [7], a moderate change in assay variability could greatly influence the result. Our first robustness analysis, which focused on random variation (not bias) on the single biomarker level, denoted a diminishing performance of IP-MS A $342 / 40$ as the $\mathrm{CV}$ increased. While IP-MS A $\beta 42 / 40$ was the best performing biomarker, random variations $\sim 5 \%$ lowered the accuracy below GFAP in CU participants and p-tau181 in CI participants. As the CV increased to $15 \%$, an accepted level of intra-assay variation in clinical chemistry, IP-MS A $\beta 42 / 40$ produced AUC's only around $60 \%$ to predict A $\beta$-positivity. In contrast, GFAP and p-tau181 maintained the same level of accuracy regardless of intraassay variation. This demonstrates that plasma measures A $\beta 42 / 40$ need to have a very low-level variability in order to maintain maximum accuracy. Given the more complex nature of IP-MS protocol and heterogeneous sample collections, we feel that an analytical variability of $10 \%$ or higher is likely across laboratories, particularly in ad hoc sampling in routine testing. While simulated variations showed clear shifts of best performance for single biomarkers, models incorporating biomarker combinations were more robust, remaining relatively stable with greater variations-IP-MS A $\beta 42 / 40$ in combination with either GFAP (all participants) and p-tau and GFAP (CI) were relatively robust up to $20 \%$. Again, however, in $\mathrm{CU}$, where IP-MS A $\beta 42 / 40$ alone was the best biomarker, higher variability affects this model selection, opting for GFAP at $>10 \% \mathrm{CV}$.

Despite both being antibody-based assays, the Simoa and IP-MS A $\beta$ assays have somewhat different biochemical properties. However, it is unknown if these technical differences contribute to the observed performances. The Simoa assay utilizes the same principle as a sandwich immunoassay, where the target analyte is first bound by a capture antibody and this immunocomplex further refined by binding of a detection antibody following washing steps to remove unspecific binding. In the Simoa $A \beta 40$ and $A \beta 42$ assays, the same capture antibody common to both analytes is used while antibodies specific to either peptide are used for detection [34]. The $A \beta 40$ and A 342 assays in the Simoa Neuro 4-plex E kit and Advantage kit are based on the same biochemical principle except that (1) different $A \beta$ antibodies are used in either kit, and (2) the latter kit provides multiplexing advantages that allow $A \beta 40$ and $A \beta 42$ to be measured alongside NfL and GFAP concurrently in the same sample. The IP-MS assay enriches for $A \beta$ in plasma by precipitating the analyte signal by binding to an $A \beta$-specific antibody or a cocktail of $A \beta$ antibodies coated onto paramagnetic beads. Following elution of the bound analytes, the signal is read with a mass spectrometer, using labeled synthetic peptides as quantification [32] standards. The biochemistry of different plasma $A \beta$ assays have been summarized in a recent review [32].

\section{Limitations}

The foremost constraint in this study is that the sample size of the ADNI participants with all plasma biomarkers was limited (total, $n=118 ; \mathrm{CU}, n=50 ; \mathrm{CI}, n=68$ ), which could have led to slightly reduced overall biomarker performance. Furthermore, it is known that preanalytical procedures and protocol variations may affect biomarker analysis and results, and therefore, we strongly advise the replication of these findings in larger independent cohorts with these available biomarker methods. However, we are encouraged that these results are in line with developing evidence from the recent literature [30]namely, IP-MS A $\beta$ being a strong predictor of amyloidosis $[6,7]$, particularly at $\mathrm{CU}[6]$ and $\mathrm{p}$-tau181 being more important at CI [15]. In studies where IP-MS A $\beta$ has not been included, GFAP has emerged as the principal candidate for amyloidosis $[18,19,31,35]$. It must be noted that plasma p-tau217 and p-tau231 are variables not included in the ADNI cohort at this time. These additional p-tau biomarkers have both been shown to have high accuracy, together with a high fold change in AD, in determining $\mathrm{A} \beta$ pathology at both the preclinical and symptomatic phases of the disease and therefore may significantly contribute to the model selections, if available $[10,36]$.

\section{Conclusion}

In this report, utilizing participants in the ADNI database, we demonstrate that plasma $A \beta$, as indexed by IP-MS, is the simplest model that best determines $A \beta$ burden at the preclinical stage. At the symptomatic phase, IP-MS A $\beta$ in combination with GFAP and p-tau181 was found to be the simplest model with the highest accuracy. 
However, the accuracy of plasma IP-MS A $\beta 42 / 40$ to indicate $A \beta$ burden deteriorates with only a modest increase in analytical variation, which will pose as an issue in ad hoc testing in clinical routine or multicenter laboratory testing in trials. In contrast, despite lower overall accuracies, GFAP and p-tau181 are highly robust. In the absence of IP-MS A $\beta$ measures, GFAP is the best predictor of amyloidosis at the preclinical stage of $\mathrm{AD}$ and, in combination with p-tau181, best predicts amyloidosis at the symptomatic phase of the disease.

\section{Supplementary Information}

The online version contains supplementary material available at https://doi. org/10.1186/s13195-021-00942-0.

Additional file 1 : Supplementary Table 1. Best models selected in the robustness analyses (within reported CV). Supplementary Figure 1. Plasma Aß42/40 distribution by groups. Supplementary Figure 2 . Plasma Aß42/40 discriminative power. Supplementary Figure 3. Plasma biomarkers distribution by $A ß$ status.

\section{Acknowledgements}

Data collection and sharing for this project was funded by the Alzheimer's Disease Neuroimaging Initiative (ADNI) (National Institutes of Health Grant U01 AG024904) and DOD ADNI (Department of Defense award number W81XWH-12-2-0012). ADNI is funded by the National Institute on Aging, the National Institute of Biomedical Imaging and Bioengineering, and through generous contributions from the following: AbbVie, Alzheimer's Association; Alzheimer's Drug Discovery Foundation; Araclon Biotech; BioClinica, Inc:; Biogen; Bristol-Myers Squibb Company; CereSpir, Inc.; Cogstate; Eisai Inc.; Elan Pharmaceuticals, Inc.; Eli Lilly and Company; Eurolmmun; F. Hoffmann-La Roche Ltd and its affiliated company Genentech, Inc;; Fujirebio; GE Healthcare; IXICO Ltd.; Janssen Alzheimer Immunotherapy Research \& Development, LLC.; Johnson \& Johnson Pharmaceutical Research \& Development LLC.; Lumosity Lundbeck; Merck \& Co., Inc.; Meso Scale Diagnostics, LLC.; NeuroRx Research; Neurotrack Technologies; Novartis Pharmaceuticals Corporation; Pfizer Inc.; Piramal Imaging; Servier; Takeda Pharmaceutical Company; and Transition Therapeutics. The Canadian Institutes of Health Research is providing funds to support ADNI clinical sites in Canada. Private sector contributions are facilitated by the Foundation for the National Institutes of Health (www.fnih. org). The grantee organization is the Northern California Institute for Research and Education, and the study is coordinated by the Alzheimer's Therapeutic Research Institute at the University of Southern California. ADNI data are disseminated by the Laboratory for Neuro Imaging at the University of Southern California.

\section{Authors' contributions}

$A L B, W S B, O H, H Z, K B$, and NJA participated in the design of this study. $H Z, K B$, and NJA supervised the study. ALB and WSB carried out the statistical analysis. ALB, WSB, and NJA wrote the paper. ERZ and TKK contributed to the revision of the paper. All authors read and approved the final version of the manuscript.

\section{Funding}

Open access funding provided by University of Gothenburg. KB is supported by the Swedish Research Council (\#2017-00915), the Alzheimer Drug Discovery Foundation (ADDF), USA (\#RDAPB-201809-2016615), the Swedish Alzheimer Foundation (\#AF-742881), Hjärnfonden, Sweden (\#FO2017-0243), the Swedish state under the agreement between the Swedish government and the County Councils, the ALF-agreement (\#ALFGBG-715986), the European Union Joint Program for Neurodegenerative Disorders (JPND2019-466-236), and the National Institute of Health (NIH), USA, (grant \#1R01 AG068398-01). $\mathrm{HZ}$ is a Wallenberg Scholar supported by grants from the Swedish Research Council (\#2018-02532), the European Research Council (\#681712), Swedish State Support for Clinical Research (\#ALFGBG-720931), the Alzheimer Drug Discovery Foundation (ADDF), USA (\#201809-2016862), the AD Strategic Fund and the Alzheimer's Association (\#ADSF-21-831376-C, \#ADSF-21-831381-C and \#ADSF-21-831377-C), the Olav Thon Foundation, the Erling-Persson Family Foundation, Stiftelsen för Gamla Tjänarinnor, Hjärnfonden, Sweden (\#FO2019-0228), the European Union's Horizon 2020 research and innovation program under the Marie Skłodowska-Curie grant agreement No 860197 (MIRIADE), and the UK Dementia Research Institute at UCL. WSB is supported by CAPES [88887.372371/2019-00] [88887.596742/2020-00]. ERZ receives financial support from CNPq [435642/2018-9] and [312410/2018- 2], Instituto Serrapilheira [Serra-1912-31365], Brazilian National Institute of Science and Technology in Excitotoxicity and Neuroprotection [465671/2014-4], FAPERGS/ MS/CNPq/SESRS-PPSUS [30786.434.24734.23112017], and ARD/FAPERGS [54392.632.30451.05032021]. PF receives financial support from CAPES [88887.507161/2020-00].

\section{Availability of data and materials}

The original data used in this manuscript is available for download in the ADNI database (http://adni.loni.usc.edu).

\section{Declarations}

\section{Ethical approval and consent to participate}

All enrolled participants or authorized representatives provided informed consent, approved by ADNI center's respective Institutional Review Boards, which were in accordance with the ethical standards of the institutional and/ or national research committee and with the principles of the 1964 Declaration of Helsinki.

\section{Consent for publication}

Not applicable.

\section{Competing interests}

HZ has served at scientific advisory boards for Eisai, Denali, Roche Diagnostics, Wave, Samumed, Siemens Healthineers, Pinteon Therapeutics, Nervgen, AZTherapies, and CogRx, has given lectures in symposia sponsored by Cellectricon, Fujirebio, Alzecure, and Biogen, and is a co-founder of Brain Biomarker Solutions in Gothenburg AB (BBS), which is a part of the GU Ventures Incubator Program. KB has served as a consultant, at advisory boards, or at data monitoring committees for Abcam, Axon, Biogen, JOMDD/Shimadzu. Julius Clinical, Lilly, MagQu, Novartis, Roche Diagnostics, and Siemens Healthineers, and is a co-founder of Brain Biomarker Solutions in Gothenburg AB (BBS), which is a part of the GU Ventures Incubator Program. OH has acquired research support (for the institution) from AVID Radiopharmaceuticals, Biogen, Eli Lilly, Eisai, Fujirebio, GE Healthcare, Pfizer, and Roche. In the past 2 years, he has received consultancy/speaker fees from Amylyx, Alzpath, Biogen, Cerveau, Fujirebio, Genentech, Roche, and Siemens.

\section{Author details}

'Department of Psychiatry and Neurochemistry, Institute of Neuroscience and Physiology, The Sahlgrenska Academy, University of Gothenburg, Gothenburg, Sweden. ${ }^{2}$ Translational Neuroimaging Laboratory, McGill Centre for Studies in Aging, McGill University, Montreal, Quebec, Canada. ${ }^{3}$ Graduate Program in Biological Sciences: Biochemistry, Universidade Federal do Rio Grande do Sul (UFRGS), Porto Alegre, Brazil. ${ }^{4}$ Clinical Memory Research Unit, Department of Clinical Sciences, Malmö, Lund University, Lund, Sweden. ${ }^{5}$ Memory Clinic, Skåne University Hospital, Malmö, Sweden. ${ }^{6}$ Department of Psychiatry, University of Pittsburgh, Pittsburgh, PA, USA. ${ }^{7}$ Department of Pharmacology, UFRGS, Porto Alegre, Brazil. ${ }^{8}$ Graduate Program in Biological Sciences: Pharmacology and Therapeutics, UFRGS, Porto Alegre, Brazil. ${ }^{9} \mathrm{Clinical}$ Neurochemistry Laboratory, Sahlgrenska University Hospital, Mölndal, Sweden. ${ }^{10}$ Department of Neurodegenerative Disease, UCL Institute of Neurology, London, UK. ${ }^{11}$ UK Dementia Research Institute at UCL, London, UK. ${ }^{12}$ Hong Kong Center for Neurodegenerative Diseases, Hong Kong, China. ${ }^{13}$ Wallenberg Centre for Molecular and Translational Medicine, Department of Psychiatry and Neurochemistry, Institute of Neuroscience and Physiology, the Sahlgrenska Academy at the University of Gothenburg, Gothenburg, Sweden. ${ }^{14}$ King's College London, Institute of Psychiatry, Psychology \& Neuroscience, Maurice Wohl Clinical Neuroscience Institute, London, UK. ${ }^{15} \mathrm{NIHR}$ Biomedical Research Centre for Mental Health \& Biomedical Research Unit for Dementia at South London \& Maudsley NHS Foundation, London, UK. 
Received: 4 August 2021 Accepted: 17 November 2021

Published online: 07 February 2022

\section{References}

1. Jack CR Jr, et al. NIA-AA Research Framework: toward a biological definition of Alzheimer's disease. Alzheimers Dement. 2018;14:535-62. https:// doi.org/10.1016/j.jalz.2018.02.018.

2. Swanson CJ, et al. A randomized, double-blind, phase $2 \mathrm{~b}$ proof-ofconcept clinical trial in early Alzheimer's disease with lecanemab, an anti-Abeta protofibril antibody. Alzheimers Res Ther. 2021;13:80. https:// doi.org/10.1186/s13195-021-00813-8.

3. Klein G, et al. Gantenerumab reduces amyloid-beta plaques in patients with prodromal to moderate Alzheimer's disease: a PET substudy interim analysis. Alzheimers Res Ther. 2019;11:101. https://doi.org/10.1186/ s13195-019-0559-z.

4. Mintun MA, et al. Donanemab in early Alzheimer's disease. N Engl J Med. 2021;384:1691-704. https://doi.org/10.1056/NEJMoa2100708.

5. Sevigny J, et al. The antibody aducanumab reduces Abeta plaques in Alzheimer's disease. Nature. 2016;537:50-6. https://doi.org/10.1038/natur e19323.

6. Nakamura A, et al. High performance plasma amyloid-beta biomarkers for Alzheimer's disease. Nature. 2018;554:249-54. https://doi.org/10.1038/ nature25456.

7. Schindler SE, et al. High-precision plasma beta-amyloid 42/40 predicts current and future brain amyloidosis. Neurology. 2019;93:e1647-59. https://doi.org/10.1212/WNL.0000000000008081.

8. Keshavan A, et al. Population-based blood screening for preclinical Alzheimer's disease in a British birth cohort at age 70. Brain. 2021;144:43449. https://doi.org/10.1093/brain/awaa403.

9. Mattsson-Carlgren N, et al. Soluble P-tau217 reflects amyloid and tau pathology and mediates the association of amyloid with tau. EMBO Mol Med. 2021;13:e14022. https://doi.org/10.15252/emmm.202114022.

10. Ashton NJ, et al. Plasma p-tau231: a new biomarker for incipient Alzheimer's disease pathology. Acta Neuropathol. 2021;141:709-24. https://doi. org/10.1007/s00401-021-02275-6.

11. Janelidze $S$, et al. Plasma P-tau181 in Alzheimer's disease: relationship to other biomarkers, differential diagnosis, neuropathology and longitudinal progression to Alzheimer's dementia. Nat Med. 2020;26:379-86. https:// doi.org/10.1038/s41591-020-0755-1.

12. Lantero Rodriguez J, et al. Plasma p-tau181 accurately predicts Alzheimer's disease pathology at least 8 years prior to post-mortem and improves the clinical characterisation of cognitive decline. Acta Neuropathol. 2020;140:267-78. https://doi.org/10.1007/s00401-020-02195-x.

13. Karikari TK, et al. Blood phosphorylated tau 181 as a biomarker for Alzheimer's disease: a diagnostic performance and prediction modelling study using data from four prospective cohorts. Lancet Neurol. 2020;19:422-33. https://doi.org/10.1016/S1474-4422(20)30071-5.

14. Thijssen $\mathrm{EH}$, et al. Diagnostic value of plasma phosphorylated tau181 in Alzheimer's disease and frontotemporal lobar degeneration. Nat Med. 2020;26:387-97. https://doi.org/10.1038/s41591-020-0762-2.

15. Karikari TK, et al. Diagnostic performance and prediction of clinical progression of plasma phospho-tau181 in the Alzheimer's Disease Neuroimaging Initiative. Mol Psychiatry. 2021;26:429-42. https://doi.org/10. 1038/s41380-020-00923-z.

16. Simren J, et al. The diagnostic and prognostic capabilities of plasma biomarkers in Alzheimer's disease. Alzheimers Dement. 2021. https://doi. org/10.1002/alz.12283.

17. Benedet AL, Milà-Alomà M, Vrillon A, et al. Differences Between Plasma and Cerebrospinal Fluid Glial Fibrillary Acidic Protein Levels Across the Alzheimer Disease Continuum. JAMA Neurol. 2021;78(12):1471-83. https://doi.org/10.1001/jamaneurol.2021.3671.

18. Chatterjee $P$, et al. Plasma glial fibrillary acidic protein is elevated in cognitively normal older adults at risk of Alzheimer's disease. Transl Psychiatry. 2021;11:27. https://doi.org/10.1038/s41398-020-01137-1.

19. Pereira JB, et al. Plasma GFAP is an early marker of amyloid-beta but not tau pathology in Alzheimer's disease. JAMA Neurol. 2021:e213671. https://doi.org/10.1001/jamaneurol.2021.3671.

20. Mattsson N, Andreasson U, Zetterberg H, Blennow K. \& Alzheimer's Disease Neuroimaging, I. Association of plasma neurofilament light with neurodegeneration in patients with alzheimer disease. JAMA Neurol. 2017;74:557-66. https://doi.org/10.1001/jamaneurol.2016.6117.

21. Ashton NJ, et al. Increased plasma neurofilament light chain concentration correlates with severity of post-mortem neurofibrillary tangle pathology and neurodegeneration. Acta Neuropathol Commun. 2019;7:5. https://doi.org/10.1186/s40478-018-0649-3.

22. Preische $\mathrm{O}$, et al. Serum neurofilament dynamics predicts neurodegeneration and clinical progression in presymptomatic Alzheimer's disease. Nat Med. 2019:25:277-83. https://doi.org/10.1038/s41591-018-0304-3.

23. Ashton NJ, et al. A multicentre validation study of the diagnostic value of plasma neurofilament light. Nat Commun. 2021;12:3400. https://doi.org/ 10.1038/s41467-021-23620-z.

24. Wihersaari L, et al. Neurofilament light as an outcome predictor after cardiac arrest: a post hoc analysis of the COMACARE trial. Intensive Care Med. 2021;47:39-48. https://doi.org/10.1007/s00134-020-06218-9.

25. Petersen RC, et al. Alzheimer's Disease Neuroimaging Initiative (ADNI): clinical characterization. Neurology. 2010;74:201-9. https://doi.org/10. 1212/WNL.0b013e3181cb3e25.

26. Landau SM, et al. Comparing positron emission tomography imaging and cerebrospinal fluid measurements of beta-amyloid. Ann Neurol. 2013;74:826-36. https://doi.org/10.1002/ana.23908.

27. Royse SK, et al. Validation of amyloid PET positivity thresholds in centiloids: a multisite PET study approach. Alzheimers Res Ther. 2021;13:99. https://doi.org/10.1186/s13195-021-00836-1.

28. John J, Dziak Donna L, Coffman Stephanie T, Lanza Runze, Li Lars S, Jermiin. Sensitivity and specificity of information criteria. Briefings in Bioinformatics. 2019, 2020;21(2):553-65. https://doi.org/10.1093/bib/bbz016.

29. West T, et al. A blood-based diagnostic test incorporating plasma Abeta42/40 ratio, ApoE proteotype, and age accurately identifies brain amyloid status: findings from a multi cohort validity analysis. Mol Neurodegener. 2021;16:30. https://doi.org/10.1186/s13024-021-00451-6.

30. Janelidze $S$, et al. Detecting amyloid positivity in early Alzheimer's disease using combinations of plasma Abeta42/Abeta40 and p-tau. Alzheimers Dement. 2021. https://doi.org/10.1002/alz.12395.

31. Verberk IMW, et al. Combination of plasma amyloid beta(1-42/1-40) and glial fibrillary acidic protein strongly associates with cerebral amyloid pathology. Alzheimers Res Ther. 2020;12:118. https://doi.org/10.1186/ s13195-020-00682-7.

32. Ashton NJ, et al. The validation status of blood biomarkers of amyloid and phospho-tau assessed with the 5-phase development framework for AD biomarkers. Eur J Nucl Med Mol Imaging. 2021;48:2140-56. https://doi. org/10.1007/s00259-021-05253-y.

33. Ashton NJ, Suárez-Calvet M, Karikari TK, Lantero-Rodriguez J, Snellman A, Sauer M, Simrén J, Minguillon C, Fauria K, Blennow K, Zetterberg H. Effects of pre-analytical procedures on blood biomarkers for Alzheimer's pathophysiology, glial activation, and neurodegeneration. Alzheimers Dement (Amst). 2021;13(1):e12168. https://doi.org/10.1002/dad2.12168.

34. Janelidze $S$, et al. Plasma beta-amyloid in Alzheimer's disease and vascular disease. Sci Rep. 2016;6:26801. https://doi.org/10.1038/srep26801.

35. Cicognola C, et al. Plasma glial fibrillary acidic protein detects Alzheimer pathology and predicts future conversion to Alzheimer dementia in patients with mild cognitive impairment. Alzheimers Res Ther. 2021;13:68 https://doi.org/10.1186/s13195-021-00804-9.

36. Palmqvist $\mathrm{S}$, et al. Discriminative accuracy of plasma phospho-tau217 for Alzheimer disease vs other neurodegenerative disorders. Jama. 2020;324:772-81. https://doi.org/10.1001/jama.2020.12134.

\section{Publisher's Note}

Springer Nature remains neutral with regard to jurisdictional claims in published maps and institutional affiliations. 